\title{
All-fiber source and sorter for multimode correlated photons
}

\author{
Kfir Sulimany $\mathbb{D}^{1}$ and Yaron Bromberg $\mathbb{D}^{1 凶}$
}

Photons occupying multiple spatial modes hold a great promise for implementing high-dimensional quantum communication. We use spontaneous four-wave mixing to generate multimode photon pairs in a few-mode fiber. We show the photons are correlated in the fiber mode basis using an all-fiber mode sorter. Our demonstration offers an essential building block for realizing highdimensional quantum protocols based on standard, commercially available fibers, in an all-fiber configuration.

npj Quantum Information (2022)8:4; https://doi.org/10.1038/s41534-021-00515-x

\section{INTRODUCTION}

High-dimensional quantum bits hold great potential for quantum communication owing to their robustness to a realistic noisy environment ${ }^{1-3}$. Implementations based on encoding information in the transverse spatial modes of photons are especially promising due to the large Hilbert space they $\operatorname{span}^{4,5}$. In recent years, such implementations were successfully demonstrated in free-space ${ }^{6,7}$. Meanwhile, efforts for multimode fiber-based technologies are expected to achieve high-dimensional quantum communication without a line of sight, based on existing multimode fiber components and infrastructures ${ }^{8-14}$.

The leading approach for generating entangled photons in transverse spatial modes is through spontaneous parametric down-conversion in bulk crystals ${ }^{15}$. However, it is extremely challenging to couple transverse entangled photons to fibers since it requires a precise mapping between the free-space transverse modes and the fiber's guided modes. Indeed, most demonstrations of distributing spatially entangled photons with fibers are limited to the coupling of only two guided modes ${ }^{8-11}$. Recently, the distribution of a photon which is entangled in six spatial modes over a 2 meter-long fiber ${ }^{12}$, and in three spatial modes over a $1-\mathrm{km}$-long fiber were demonstrated ${ }^{13}$. However, these methods require accurate calibrations, limiting implementations in real-life scenarios.

An alternative for coupling free-space entangled photons to fiber is to generate the photons inside the fiber by using spontaneous four-wave mixing (SFWM). Over the past two decades, generation of photon pairs by SFWM was studied using multiple types of single-mode optical fibers ${ }^{16}$, including photonic crystal fibers ${ }^{17-20}$, dispersion-shifted fibers ${ }^{21-23}$, and birefringent fibers ${ }^{24,25}$. SFWM in multimode fibers was recently utilized for generating photons occupying a high-dimensional transverse mode ${ }^{26-28}$. Generating photon pairs in a superposition of multiple fiber modes requires a precise analysis of the phase-matching conditions that will allow multiple SFWM processes in the same spectral channel ${ }^{29-32}$. These theoretical works predict that the photon pair sources can be tunable over a wide range of wavelengths, from the ultraviolet to the infrared and the telecommunication range. Experimentally, such phase-matching conditions were recently studied for parametric amplification of weak signals ${ }^{33}$, but not in the spontaneous regime. Hence correlations between pairs of photons generated in multiple fiber modes were not measured to date.

In this work, we propose and demonstrate a fiber source of photon pairs, which occupy multiple fiber modes. Our measurements prove that the photons are correlated in the guided mode basis, by mapping the modes the photons occupy to their arrival times at the end of a 1-km-long fiber. The 1-km fiber acts as an all-fiber in-line mode sorter, in contrast to bulk free-space mode sorters that are typically used for measuring correlations between transverse modes $^{15,34-36}$. Our in-line mode sorting configuration allows us to measure the two-dimensional (2D) histogram of the arrival times of the photons, which reveals that the photons occupy three guided modes of the fiber. By analyzing the histogram we achieve the twophoton modal decomposition and verify the spatial correlations of photon pairs generated in the multimode fiber.

\section{RESULTS}

\section{Multimode correlated photons source}

Our source is based on coupling Ti:Sapphire mode-locked pulses (pulse duration $140 \mathrm{fs}$, wavelength $\lambda_{\text {pump }}=695 \mathrm{~nm}$ ) into a fewmode fiber as shown in Fig. 1. In SFWM, two pump photons are spontaneously annihilated, and two photons called signal and idler are generated in two spectral channels $\left(\lambda_{s}=542 \mathrm{~nm}, \lambda_{i}=\right.$ $970 \mathrm{~nm}$ ). Each spectral channel is composed of many different spatial modes. The photons occupy the guided modes of the fiber, which can be approximated by the linearly polarized (LP) modes of a weakly guiding optical fiber. The state of the photons is determined by the phase-matching conditions and can be written as: $|\Psi\rangle=a\left|L P_{02}\right\rangle_{s}\left|L P_{01}\right\rangle_{i}+\beta\left|L P_{11}\right\rangle_{s}\left|L P_{11}\right\rangle_{i}$ where subscripts $s \quad$ (i) mark the mode of the signal (idler) photon and the coefficients $a$, $\beta$ are determined by the nonlinear overlap integral (see Supplementary Equation 3). The term $\left|L P_{01}\right\rangle_{s}\left|L P_{02}\right\rangle_{i}$ is not present in the quantum state as the mode $L P_{02}$ is not guided in our fiber for the wavelength of the idler photon. The extension of this scheme to higher dimensions and other spectral bands is presented in Supplementary Note 1.

The photon pairs are generated mostly in the first few tens of centimeters of the fiber, after which the peak power of the pump pulse is too weak for SFWM due to its temporal spreading (see Supplementary Note 2 for more information). To quantify the efficiency of the pair generation we use a $20 \mathrm{~cm}$ section of SMF-28 to measure the coincidence detection rate as a function of the pump average power, exhibiting a quadratic scaling as expected for a four-wave mixing process (Fig. 1b). The coincidence to the accidental ratio we obtain for a pump average power of $10 \mathrm{~mW}$ is 850 (see Supplementary Note 3 for more details). In principle, to improve the coincidence rate we could use higher pump powers. Increasing the pump power, however, will also increase parasitic

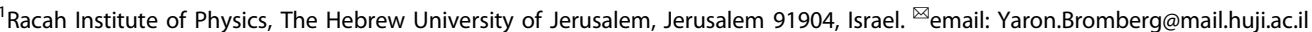




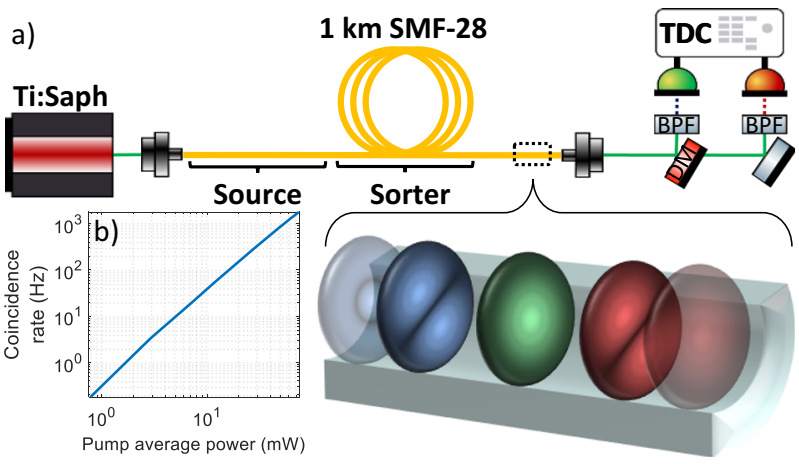

Fig. 1 An all-fiber multimode source and mode sorter for photon pairs correlated in the fiber modes. a Ultrashort pulses of $140 \mathrm{fs}$ $\left(\lambda_{\text {pump }}=695 \mathrm{~nm}\right)$ are coupled into a 1-km-long fiber. Pump photons are spontaneously annihilated and pairs of signal and idler photons are generated at two different spectral channels $\left(\lambda_{s}=542 \mathrm{~nm}, \lambda_{i}=\right.$ $970 \mathrm{~nm}$ ). At these wavelengths, the fiber (SMF-28) supports a few modes, where the modal distribution of the photon pairs is determined by the phase-matching condition of the fiber. After the first few tens of centimeters, the temporal spread of the pump pulse prevents SFWM. In the next 1-km of the fiber, the different modes are separated due to modal dispersion (inset). Higher spatial modes arrive after lower spatial modes, and shorter wavelengths arrive after longer wavelengths. At the output of the fiber, the signal and idler photons are spectrally separated by a dichroic mirror (DM), filtered by a bandpass filter (BPF), and their arrival times are registered using two single-photon detectors and a time-to-digital converter (TDC). An electronic delay of 70ns is introduced to the idler detector to compensate for the chromatic delay between the signal and idler photons. b Experimentally measured coincidence rate as a function of the pump average power for a $20-\mathrm{cm}$-long fiber, exhibiting a quadratic scaling.

Raman scattering. In our system, Raman scattering hardly adds noise since it is temporally separated from the generated photon pairs. However, the pump power is limited since the photon counts due to Raman scattering exceed the maximal count rate of our detectors $(\approx 5 \mathrm{Mhz})$. This limitation can be circumvented by using superconducting nanowire detectors with an order of magnitude higher maximal count rates ( $\approx 50 \mathrm{Mhz})$, or by using inline fiber Bragg gratings to filter the pump light before the sorter so that the pump will not generate Raman scattering along the 1km-fiber.

\section{Multimode photons sorter}

Next, we use a $1 \mathrm{~km}$ section of the same fiber, which serves as a photon pairs source and as a mode sorter of the fiber's guided modes. Due to modal Group Delay Dispersion (GDD), the arrival times of the photons at the end of the fiber depend on their modal distribution and their spectral channel, as depicted in Fig. 1. We can therefore map the arrival times of the photons to their modal decomposition, up to modal degeneracy in symmetric fiber cores. Although this sorting scheme is quite common in classical optics $^{37}$, it was only recently demonstrated at the single-photon level for weak coherent pulses ${ }^{38}$. Here we use the same principle for entangled photons. In our setup, the temporal resolution is limited by the jitter of the avalanche photodiodes which is $400 \mathrm{ps}$. Since the GDD of our fiber is on the scale of $1 \mathrm{~ns} / \mathrm{km}$, a $1-\mathrm{km}$-long fiber is sufficient to temporally separate the modes.

\section{Two-photon modal distribution measurement}

To investigate the modal distribution of the two-photon state, we use the mode-to-time mapping and study the temporal two-photon probability $P\left(T_{s}, T_{i}\right)$ that describes the probability to detect a signal photon at time $T_{s}$ and an idler photon at time $T_{i}$. To this end, we plot
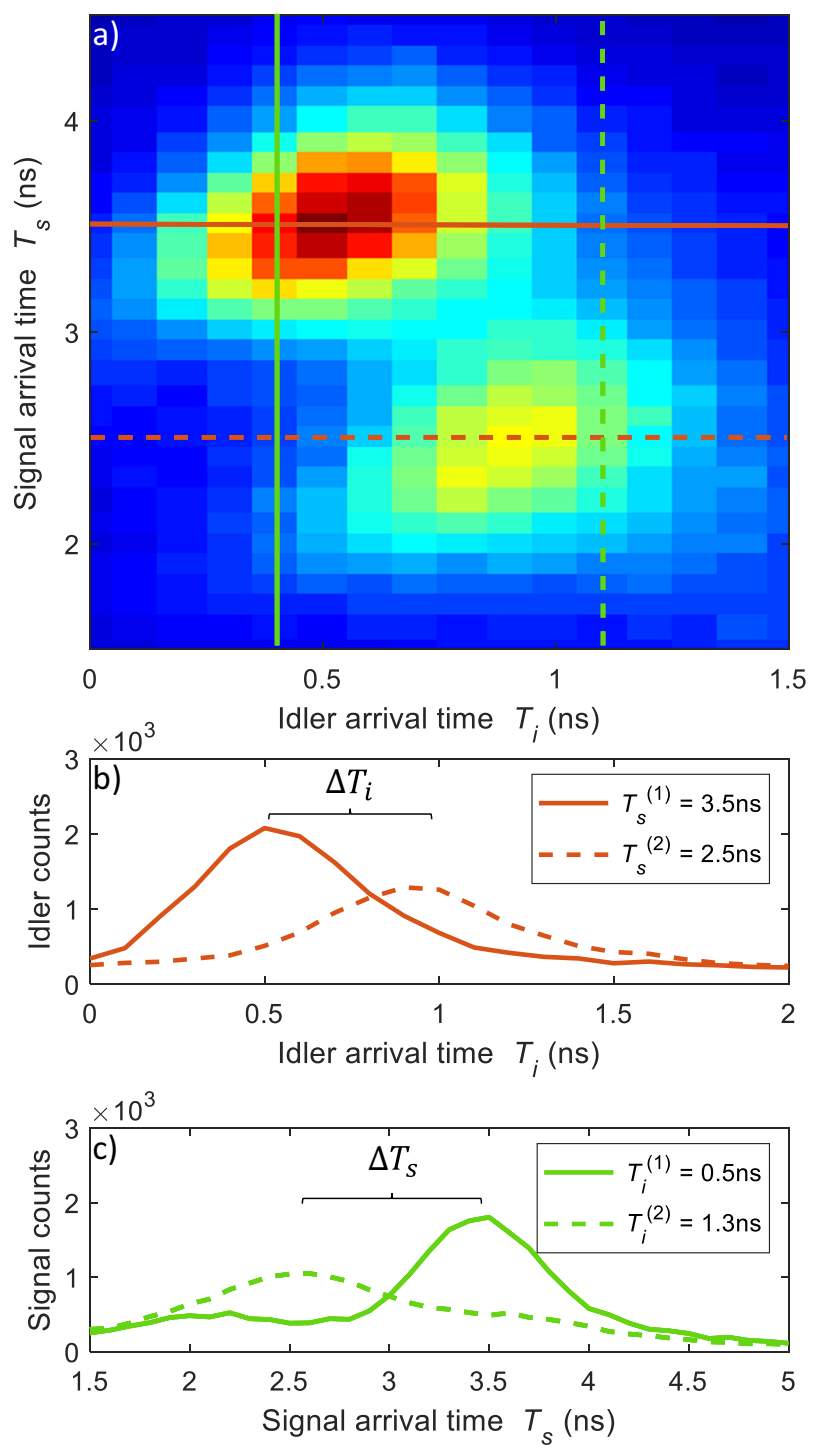

Fig. 2 Temporal two-photon probability. a Histogram of the arrival times $T_{s}, T_{i}$ of the signal, and idler photons. The arrival times are measured relative to an electronic trigger from the pump laser which serves as a global clock, and after adding an electronic delay of $70 \mathrm{~ns}$ to the idler detector to compensate for the chromatic delay between the signal and idler photons. The two off-diagonal peaks indicate that the two-photon state is not separable. We, therefore, conclude that the photons are correlated in the modal basis. The two peaks correspond to the occupation of modes $\left|L P_{02}\right\rangle_{s}\left|L P_{01}\right\rangle_{i}$ and $\left|L P_{11}\right\rangle_{s}\left|L P_{11}\right\rangle_{i}$, as verified by numerical computation of the fiber's modal group delays. b, c Cross-sections of the two-dimensional histogram along the lines marked in (a), emphasizing the modal correlations. For example, post selecting events with an idler's arrival time of $T_{i}^{(1)}=0.4 \mathrm{~ns}$ (green solid curve) shows localization of the signal photon at $T_{s}^{(2)}=3.5 \mathrm{~ns}$. In (b) the post-selection is on the signal photon, while in (c) it is on the idler photon. The measured delay between the two peaks is $\Delta T_{s}=1 \mathrm{~ns}$ for the signal photons and $\Delta T_{i}=0.5 \mathrm{~ns}$ for the idler photons.

the two-dimensional histogram of the arrival times after compensating for chromatic dispersion (Fig. 2a). Two correlation peaks are observed, corresponding to the delay between either $\left|L P_{02}\right\rangle_{s}$ and $\left|L P_{01}\right\rangle_{i}$ or between $\left|L P_{11}\right\rangle_{s}$ and $\left|L P_{11}\right\rangle_{i}$. Clearly, the two-photon probability is not-separable, indicating that photons are correlated in the modal basis. To quantify the correlation of the two photons we post select two arrival times for the signal $\left(T_{s}^{(1)}, T_{s}^{(2)}\right)$ and two arrival 
times for the idler $\left(T_{i}^{(1)}, T_{i}^{(2)}\right)$. The post-selected arrival times are chosen to maximize the Pearson correlation coefficient: $P C C=$ $\sum_{k=1}^{2} \sum_{l=1}^{2} P\left(T_{s}^{(k)}, T_{i}^{(l)}\right)\left(T_{s}^{(k)}-\mu_{T_{s}}\right)\left(T_{i}^{(l)}-\mu_{T_{i}}\right) /\left(\sigma_{T_{s}} \sigma_{T_{i}}\right) \quad$ where $\mu_{T_{s}}, \mu_{T_{i}}$ are the mean arrival times of the signal and idler photons and $\sigma_{T_{s}}, \sigma_{T_{i}}$ are their standard deviations. We obtain $P C C=0.51 \pm$ 0.012 , which indicates a strong correlation. The main source of correlation degradation in our system is the $400 \mathrm{ps}$ jitter of the detectors, which causes circular smearing of the histogram peaks. Another source of decorrelation is the uncertainty in the creation times of the pairs, which results in a diagonal spread of about $\approx 200$ ps that hardly effects the PCC between the chosen arrival times. In principle, the intermodal coupling can also add decorrelation, however, the PCC is sensitive only to mode mixing that occurs in the first few tens of centimeters of the fiber because the arrival times of photons which experience mode coupling after a longer distance will be different from the post-selected times $\left(T_{s}^{(1)}, T_{s}^{(2)}\right),\left(T_{i}^{(1)}, T_{i}^{(2)}\right)$. Thus PCC degradation due to intermodal mode mixing, which is typically on the order of $20 \mathrm{db} / \mathrm{km}^{39}$, is negligible.

\section{Modal group delay simulation}

To show that the measured delays between the signal and idler photons match the expected delays for an SMF-28 fiber, we numerically calculated its modal group delays. We solve the scalar wave equation for an SMF-28 fiber, with a 4.2 um core radius, corecladding index difference of $\Delta=0.33 \%$, and a step-index profile with a typical dip shape. The modal delay of $L P_{11}, L P_{02}$ modes, relative to the fundamental mode is presented in Fig. 3 . We chose the fundamental mode as a reference to cancel the chromatic dispersion. At the signal's wavelength, the delay between the $L P_{02}$ and $L P_{11}$ is $\Delta T_{s}=1 \mathrm{~ns}$. At the idler's wavelength, the delay of $L P_{01}$ and $L P_{11}$ is $\Delta T_{i}=0.5 \mathrm{~ns}$. These delays are in agreement with the temporal correlations found experimentally, supporting the modeto-time mapping scheme (Fig. 1b).

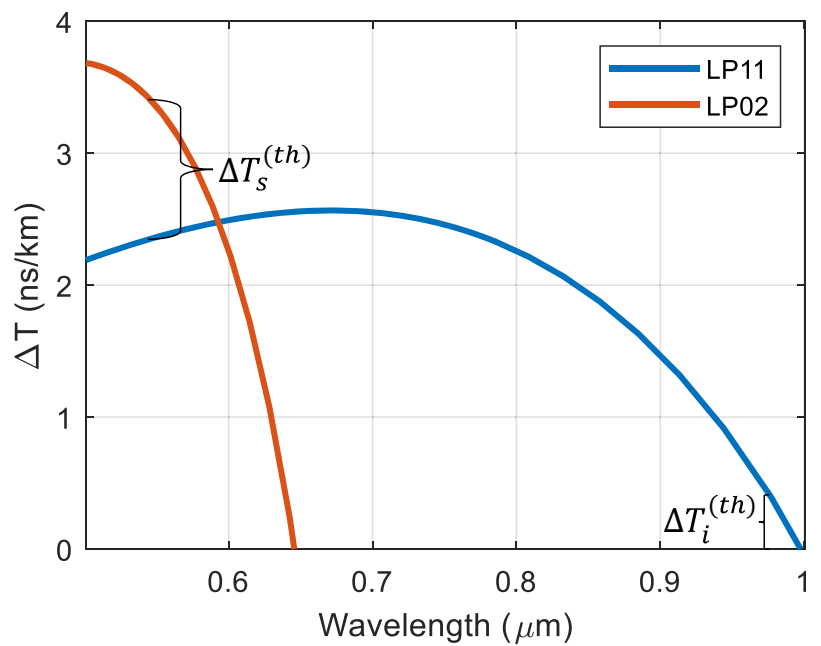

Fig. 3 Numerical computation of the modal delays of the $L P_{11}$ (blue curve) and the $L P_{02}$ (red curve) modes. The delays are presented relative to the fundamental mode $L P_{01}$, to compensate for the chromatic delay. For the signal photon at $\lambda_{s}=542 \mathrm{~nm}$, the delay between the $L P_{02}$ and $L P_{11}$ modes is $\Delta T_{s}^{(t h)}=1 \mathrm{~ns} / \mathrm{km}$, in agreement with the experimentally measured delays presented in Fig. 2c. For the idler photon at $\lambda_{i}=970 \mathrm{~nm}$, the delay between the $L P_{11}$ and $L P_{01}$ modes is $\Delta T_{i}^{(t h)}=0.5 \mathrm{~ns} / \mathrm{km}$, in agreement with measured delays reported in Fig. $2 b$.

\section{DISCUSSION}

In conclusion, we have demonstrated generation and sorting of correlated photon pairs occupying high-order modes of a commercially available fiber. The all-fiber configuration opens the door for implementing high-dimensional photonic quantum bits in fiber-based applications. For example, the mode-to-time mapping can potentially solve the challenge of scaling the number of required detectors with the number of fiber modes, an outstanding challenge in conventional mode sorters. Towards this end, it is necessary to improve the temporal resolution of the system, for example by using superconducting nanowire singlephoton detectors with jitter times as low as a few picoseconds, and faster electronics. It will allow sorting more transverse modes and using shorter fibers for the temporal mode sorter, which in turn will decrease the background noise caused by fluorescence and parasitic nonlinear processes in the fiber.

In order to manipulate the photons coherently and apply projective measurements in two mutually unbiased bases one can use multi-plane light converters (MPLC) ${ }^{34,35,40,41}$. We note that by combining the all-fiber temporal sorter with an all-fiber wavefront modulator that we recently developed ${ }^{42}$, it would be possible to demonstrate an all-fiber sorter on a mutually unbiased basis, opening the door for all-fiber quantum communication protocols with high-dimensional quantum bits.

Addressing these challenges will allow exploring applications of the all-fiber source and sorter. For example, using an in-line multimode fiber beam splitter one could split the photon pairs and route each photon to a different remote user. Such configuration is relevant for device-independent quantum key distribution, where an untrusted user (Charlie) distributes entangled photon pairs to Alice and Bob, who generate a secure key based on Bell measurements ${ }^{43}$. A more immediate application of the all-fiber source is quantum communication protocols that rely on sending both photons to the same target. Examples include quantum dense coding ${ }^{44,45}$, high capacity quantum key distribution ${ }^{46,47}$, and direct quantum communication ${ }^{48}$.

\section{METHODS}

\section{Experimental setup}

An optical fiber (SMF-28) is pumped by a Ti:Sapphire laser (Coherent Chameleon Ultra II, $680-1060 \mathrm{~nm}, 140 \mathrm{fs}$ duration, $80 \mathrm{MHz}$ repetition rate). Before coupling to the fiber, the laser was filtered using a bandpass filter (Thorlabs FB700-40). The signal and idler photons were separated using a dichroic mirror (DM) with an edge at $925 \mathrm{~nm}$ (Semrock FF925-Di01). In each arm, the pump beam was blocked using spectral filters. In the signal arm, we employed a short-pass filter (Semrock BSP01-633R), and a bandpass filter (Semrock FF01-540). In the Idler arm, we employed a longpass filter (Semrock BLP01-808R) and a bandpass filter (Semrock LL01-976). The signal and idler photons were coupled into two optical fibers (SMF-28) and detected using avalanche photodetectors (Excelitas SPCM-AQ4C), with a quantum efficiency of $50 \%$ for the signal photons and $15 \%$ for the idler photons. The arrival times of the photons were registered using a time-todigital converter (Swabian Time Tagger 20).

\section{DATA AVAILABILITY}

The data that support the findings of this study are available from the corresponding author upon reasonable request.

Received: 9 May 2021; Accepted: 9 November 2021; Published online: 14 January 2022

\section{REFERENCES}

1. Cerf, N. J., Bourennane, M., Karlsson, A. \& Gisin, N. Security of quantum key distribution using d-level systems. Phys. Rev. Lett. 88, 127902 (2002).

2. Erhard, M., Krenn, M., \& Zeilinger, A. Advances in high-dimensional quantum entanglement. Nat. Rev. Phys. 2, 365-381 (2020). 
3. Cozzolino, D., Da Lio, B., Bacco, D. \& Oxenløwe, L. K. High-dimensional quantum communication: benefits, progress, and future challenges. Adv. Quantum Technol. 2, 1900038 (2019a).

4. Molina-Terriza, G., Torres, J. P. \& Torner, L. Twisted photons. Nat. Phys. 3, 305 (2007).

5. Erhard, M., Fickler, R., Krenn, M. \& Zeilinger, A. Twisted photons: new quantum perspectives in high dimensions. Light Sci. Appl. 7, 17146 (2018).

6. Krenn, M., Handsteiner, J., Fink, M., Fickler, R. \& Zeilinger, A. Twisted photon entanglement through turbulent air across vienna. Proc. Natl Acad. Sci. USA 112, 14197 (2015).

7. Sit, A. et al. High-dimensional intracity quantum cryptography with structured photons. Optica 4, 1006 (2017).

8. Löffler, W. et al. Fiber transport of spatially entangled photons. Phys. Rev. Lett. 106, 240505 (2011).

9. Kang, Y. et al. Measurement of the entanglement between photonic spatial modes in optical fibers. Phys. Rev. Lett. 109, 020502 (2012).

10. Cozzolino, D. et al. Air-core fiber distribution of hybrid vector vortex-polarization entangled states. Adv. Photonics 1, 046005 (2019b).

11. Alarcon, A. et al. Few-mode-fiber technology fine-tunes losses in quantum communication systems, Phys. Rev. Appl. 16, 034018 (2021).

12. Valencia, N. H., Goel, S., McCutcheon, W., Defienne, H., \& Malik, M., Unscrambling entanglement through a complex medium, Nat. Phys. 16, 1112-1116 (2020).

13. Cao, $\mathrm{H}$. et al. Distribution of high-dimensional orbital angular momentum entanglement over a $1 \mathrm{~km}$ few-mode fiber. Optica 7, 232 (2020).

14. Zhou, Y. et al. High-fidelity spatial mode transmission through a 1-km-long multimode fiber via vectorial time reversal. Nat. Commun. 12, 1 (2021).

15. Mair, A., Vaziri, A., Weihs, G. \& Zeilinger, A. Entanglement of the orbital angular momentum states of photons. Nature 412, 313 (2001).

16. Park, K., Lee, D., Boyd, R. W., \& Shin, H. Telecom c-band photon-pair generation using standard smf-28 fiber. Opt. Commun. 484, 126692 (2020).

17. Sharping, J. E., Chen, J., Li, X., Kumar, P. \& Windeler, R. S. Quantum-correlated twin photons from microstructure fiber. Opt. Express 12, 3086 (2004).

18. Rarity, J., Fulconis, J., Duligall, J., Wadsworth, W. \& Russell, P. S. J. Photonic crystal fiber source of correlated photon pairs. Opt. Express 13, 534 (2005).

19. Fan, J., Migdall, A. \& Wang, L. Efficient generation of correlated photon pairs in a microstructure fiber. Opt. Lett. 30, 3368 (2005).

20. Cohen, O., Lundeen, J. S., Smith, B. J., Puentes, G., Mosley, P. J. \& Walmsley, I. A. Tailored photon-pair generation in optical fibers. Phys. Rev. Lett. 102, 123603 (2009).

21. Li, X., Voss, P. L., Sharping, J. E. \& Kumar, P. Optical-fiber source of polarizationentangled photons in the $1550 \mathrm{~nm}$ telecom band. Phys. Rev. Lett. 94, 053601 (2005).

22. Takesue, H. \& Inoue, K. 1.5- $\mu \mathrm{m}$ band quantum-correlated photon pair generation in dispersion-shifted fiber: suppression of noise photons by cooling fiber. Opt. Express 13, 7832 (2005).

23. Dyer, S. D., Baek, B. \& Nam, S. W. High-brightness, low-noise, all-fiber photon pair source. Opt. Express 17, 10290 (2009).

24. Smith, B. J., Mahou, P., Cohen, O., Lundeen, J. \& Walmsley, I. Photon pair generation in birefringent optical fibers. Opt. Express 17, 23589 (2009).

25. Lugani, J., Francis-Jones, R. J., Boutari, J. \& Walmsley, I. A. Spectrally pure single photons at telecommunications wavelengths using commercial birefringent optical fiber. Opt. Express 28, 5147 (2020).

26. Cruz-Delgado, D. et al. Fiber-based photon-pair source capable of hybrid entanglement in frequency and transverse mode, controllably scalable to higher dimensions. Sci. Rep. 6, 27377 (2016).

27. Rottwitt, K., Koefoed, J. G. \& Christensen, E. N. Photon-pair sources based on intermodal four-wave mixing in few-mode fibers. Fibers 6, 32 (2018).

28. Guo, C., Su, J., Zhang, Z., Cui, L. \& Li, X. Generation of telecom-band correlated photon pairs in different spatial modes using few-mode fibers. Opt. Lett. 44, 235 (2019a).

29. Pourbeyram, H., \& Mafi, A., Photon pair generation with tailored frequency correlations in graded-index multimode fibers. Opt. Lett. 43, 2018-2021 (2018).

30. Rottwitt, K., Christensen, J. B., Christensen, E. N., \& Koefoed, J. G. Quantum information processing using intermodal four-wave mixing in multi-mode optical fibers. In Proc. 2019 21st International Conference on Transparent Optical Networks (ICTON) 1 (IEEE, 2019).

31. Ekici, C. \& Dinleyici, M. S. Graded-index optical fiber transverse-spatial-mode entanglement. Phys. Rev. A 102, 013702 (2020)

32. Goudreau, E. S., Kupchak, C., Sussman, B. J., Boyd, R. W. \& Lundeen, J. S. Theory of four-wave mixing of cylindrical vector beams in optical fibers. JOSA B 37, 1670 (2020).

33. Shamsshooli, A., Guo, C., Parmigiani, F., Li, X. \& Vasilyev, M. Toward generation of spatially-entangled photon pairs in a few-mode fiber. In CLEO: Applications and Technology JTh2A-27 (Optical Society of America, 2020).
34. Fontaine, N. K., Ryf, R., Chen, H., Neilson, D. T., Kim, K. \& Carpenter, J. Laguerregaussian mode sorter. Nat. Commun. 10, 1 (2019).

35. Krenn, M., Huber, M., Fickler, R., Lapkiewicz, R., Ramelow, S. \& Zeilinger, A. Generation and confirmation of a $(100 \times 100)$-dimensional entangled quantum system. Proc. Natl Acad. Sci. USA 111, 6243 (2014).

36. Berkhout, G. C., Lavery, M. P., Courtial, J., Beijersbergen, M. W. \& Padgett, M. J. Efficient sorting of orbital angular momentum states of light. Phys. Rev. Lett. 105, 153601 (2010)

37. Painchaud, Y., LeBel, P., Duguay, M. \& Black, R. J. Time-resolved identification of modes and measurement of intermodal dispersion in optical fibers. Appl. Opt. 31, 2005 (1992).

38. Chandrasekharan, H. K. et al. Observing mode-dependent wavelength-to-time mapping in few-mode fibers using a single-photon detector array. APL Photonics 5, 061303 (2020).

39. Kaliteevskiy, N., Korolev, A., Koreshkov, K., Nazarov, V. \& Sterlingov, P. Two-mode coupling model in a few mode fiber. Opt. Spectrosc. 114, 913 (2013).

40. Lib, O., Sulimany, K., \& Bromberg, Y. Reconfigurable synthesizer for quantum information processing of high-dimensional entangled photons. Preprint at arXiv:2108.02258 (2021).

41. Fontaine, N. K. et al. Hermite-gaussian mode multiplexer supporting 1035 modes. In 2021 Optical Fiber Communications Conference and Exhibition (OFC) 1-3 (IEEE, 2021).

42. Resisi, S., Viernik, Y., Popoff, S. M. \& Bromberg, Y. Wavefront shaping in multimode fibers by transmission matrix engineering. APL Photonics 5, 036103 (2020).

43. Ekert, A. K. Quantum cryptography based on bell's theorem. Phys. Rev. Lett. 67, 661 (1991).

44. Hu, X.-M., Guo, Y., Liu, B.-H., Huang, Y.-F., Li, C.-F. \& Guo, G.-C. Beating the channel capacity limit for superdense coding with entangled ququarts. Sci. Adv. 4, eaat9304 (2018)

45. Guo, Y., Liu, B.-H., Li, C.-F. \& Guo, G.-C. Advances in quantum dense coding. Adv. Quantum Technol. 2, 1900011 (2019b).

46. Cabello, A. Quantum key distribution in the holevo limit. Phys. Rev. Lett. 85, 5635 (2000).

47. Long, G.-L. \& Liu, X.-S. Theoretically efficient high-capacity quantum-keydistribution scheme. Phys. Rev. A 65, 032302 (2002).

48. Deng, F.-G., Long, G. L. \& Liu, X.-S. Two-step quantum direct communication protocol using the einstein-podolsky-rosen pair block. Phys. Rev. A 68, 042317 (2003).

\section{ACKNOWLEDGEMENTS}

The authors kindly thank Hagai Eisenberg and Avi Pe'er for many fruitful discussions and suggestions. This research was supported by the United States-Israel Binational Science Foundation (BSF) (Grant No. 2017694). K.S. and Y.B. acknowledge the support of the Israeli Council for Higher Education, the Israel National Quantum Initiative (INQI), and the Zuckerman STEM Leadership Program.

\section{AUTHOR CONTRIBUTIONS}

K.S. proposed the idea, performed the experiment, and carried out the analysis; Y.B. supervised the project.

\section{COMPETING INTERESTS}

The authors declare no competing interests.

\section{ADDITIONAL INFORMATION}

Supplementary information The online version contains supplementary material available at https://doi.org/10.1038/s41534-021-00515-x.

Correspondence and requests for materials should be addressed to Yaron Bromberg.

Reprints and permission information is available at http://www.nature.com/ reprints

Publisher's note Springer Nature remains neutral with regard to jurisdictional claims in published maps and institutional affiliations. 
Open Access This article is licensed under a Creative Commons Ac Attribution 4.0 International License, which permits use, sharing,
adaptation, distribution and reproduction in any medium or format, as long as you give appropriate credit to the original author(s) and the source, provide a link to the Creative Commons license, and indicate if changes were made. The images or other third party material in this article are included in the article's Creative Commons license, unless indicated otherwise in a credit line to the material. If material is not included in the article's Creative Commons license and your intended use is not permitted by statutory regulation or exceeds the permitted use, you will need to obtain permission directly from the copyright holder. To view a copy of this license, visit http://creativecommons. org/licenses/by/4.0/.

(c) The Author(s) 2022 